\title{
Increased Nitric Oxide Production and GFAP Expression in the Brains of Influenza A/NWS Virus Infected Mice
}

\author{
Chiako Watanabe - Hisashi Kawashima • \\ Kouji Takekuma · Akinori Hoshika • \\ Yasuo Watanabe
}

Accepted: 5 November 2007/Published online: 14 December 2007

(C) The Author(s) 2007

\begin{abstract}
The cause of influenza to the brain was investigated using the $\mathrm{A} / \mathrm{NWS} / 33$ influenza virus infected BALB/c mouse model. NOS-2 mRNA levels in the infected mouse brain was greater than in control mice in all brain regions examined, particularly in the olfactory bulb and hippocampus by 1 day p.i. On the contrary, no differences in NOS-1 or NOS-3 mRNA levels were found between infected and control mice. There was also a marked increase in the levels of metabolites of nitric oxide in the olfactory bulb and hippocampus. Immunohistochemistry showed positive staining for anti-NOS-2 primarily in the hippocampus of infected mice. Further, anti-NOS-2 and GFAP staining was mostly found around capillary blood vessels of the hippocampus starting early in the course of the disease. These results indicate that the NWS enhances the activation of astrocytes and NOS-2 expression which in turn enhances NO production and the expansion of capillary blood vessels.
\end{abstract}

Keywords Influenza A/NWS/33 virus - BALB/c mouse NO · NOS-2 - Astrocyte · Olfactory bulb · Hippocampus · Brain capillary blood vessels

C. Watanabe $(\bowtie) \cdot$ H. Kawashima $\cdot$ K. Takekuma $\cdot$ A. Hoshika Department of Pediatrics, Tokyo Medical University, 6-7-1 Nishishinjuku, Shinjuku-ku, Tokyo 160-0023, Japan

e-mail: chiako@tokyo-med.ac.jp

\section{Y. Watanabe}

Department of Pharmacology/Pharmacotherapy, School of Medicinal Pharmaceutical Sciences, Nihon Pharmaceutical University, Inamachi, Saitama 360-0806, Japan

\section{Introduction}

Influenza associated encephalopathy (IE) is one of the most severe neurological diseases amongst children, mostly under the age of 5. It is marked by rapid progression and the mortality rate is estimated to range from 27 to $44 \%$ [1]. Recently, we reported that production of the biological free radical gas, nitric oxide (NO), in the serum and cerebrospinal fluid (CSF) of patients with IE was increased in the initial stages and correlated with disease severity $[2,3]$.

Yokota et al. hypothesized that the influenza virus activates the limbic system followed by glial cell activation which in turn produces NO and inflammatory cytokines which flood the brain and cause a cytokine storm [4]. Recent in vitro studies by Imanishi et al. showed that a mouse lung-adapted strain of influenza virus could induce inducible NO synthase (NOS-2) mRNA expression and NOS-2-mediated NO production in murine macrophage cell [5]. Moreover, Ino et al. studied five autopsies cases of IE patients. They reported that four out of the five patients showed degenerated astrocytic spines in the cortex, by glial fibrillary acidic protein (GFAP) staining. They suspected that the IE was largely due to these altered glial cells (Ino et al. unpublished).

Taken together, it is likely that IE is related to both the influence of $\mathrm{NO}$ and glial cells changes. Nevertheless, there have been no reports on a relationship between NOS-2 and glial cells within the brain after infection with the influenza virus. Thus, the aim of these studies is to better understand the clinical state of IE by focusing on NO and astrocytes in the central nervous system (CNS) after influenza virus infection. By using the $\mathrm{A} / \mathrm{NWS} / 33$ influenza virus infected $\mathrm{BALB} / \mathrm{c}$ model mouse, we were able to demonstrate that influenza virus infection leads to an up regulation of NOS2 and astrocytes, mostly around capillary blood vessels of 
the hippocampus and olfactory bulb, starting at an early stage of the disease.

Although IE has been reported for more than a decade, a therapy for IE has not yet been defined. We expect that our results will provide evidence which will aid in the development of a novel IE therapy.

\section{Experimental procedures}

Virus

Influenza A/NWS virus, which is a mouse brain adapted type of human influenza A/NWS/33 virus was used. (A kind gift from Dr. K. Hayashi, Toyama University, Japan.)

\section{Experimental animals}

In total, 82 male BALB/c mice (5-week-old) were purchased from Japan Charles River Co. and given free access to food and water (Oriental Yeast, Co., Tokyo) and acclimatized for at least a week before the experiment.

\section{Anesthetics}

Each mouse was anesthetized by inhalation of ether before being inoculated intranasally with $50 \mu \mathrm{NWS}, 5.0 \times 10^{7}$ plaque-forming units $(\mathrm{PFU}) / \mathrm{ml}\left(5.0 \times 10^{5} \mathrm{PFU} /\right.$ mouse $)$. For negative control mice, the same amounts of $0.1 \mathrm{M}$ phosphate-buffered saline (PBS) were intranasally inoculated. The mice were examined at 1,3 and 6 days postinfection (p.i.).

\section{Body weight}

Body weight changes were monitored daily.

\section{Euthanasia}

All mice were decapitated whilst under ether inhalational anesthesia. Each brain was divided into eight regions (frontal cortex, occipital cortex, cerebellum, medullar oblongata, hippocampus, corpus striatum, thalamus/hypothalamus and olfactory bulb). Then each section was immediately frozen with liquid nitrogen.

$\mathrm{NO}_{2}^{-}$and $\mathrm{NO}_{3}^{-}$measurements by HPLC-UV method

Both $\mathrm{NO}_{2}$ and $\mathrm{NO}_{3}\left(\mathrm{NO}_{x}\right)$ levels in each brain region were measured by HPLC-UV system (ENO-10, EICOM) which was reported by us [3]. Briefly, thawed brain regions were deproteinized with a ten times volume of methanol and then centrifuged $\left(12,000\right.$ r.p.m. for $20 \mathrm{~min}$ at $\left.4^{\circ} \mathrm{C}\right)$ to avoid an occlusion in the polystyrene polymer column used in this system, by protein or other macromolecules. Detection was measured at $540 \mathrm{~nm}$ (absorption) and the concentrations were calculated from the area under the curve (AUC) of $\mathrm{NO}_{2}$ and $\mathrm{NO}_{3}$ (Power Chrome, EICOM, Kyoto) by Griess reaction.

\section{RNA extraction}

RNA was extracted from each part of the brain by using a QIA amp RNA extraction kit (QIAGEN K.K., Tokyo), according to the protocol suggested by the manufacturer.

cDNA synthesis

cDNA was synthesized from purified RNA by using the Transcriptor First Strand cDNA Synthesis Kit (Roche, Switzerland). Random hexamer primer was used and the protocol was as follows; after annealing for $10 \mathrm{~min}$ at $25^{\circ} \mathrm{C}$, and incubating $30 \mathrm{~min}$ at $55^{\circ} \mathrm{C}$, the reaction was heated to $85^{\circ} \mathrm{C}$ for $5 \mathrm{~min}$ and chilled on ice.

Real time reverse transcriptase-polymerase chain reaction (RT-PCR)

Messenger RNA levels of NOS-1, NOS-2 and NOS-3 were quantified by using a Light Cycler Fast Start DNA Master HybProbe (Roche). This was done according to the following protocol; Pre-incubation was $95^{\circ} \mathrm{C}$ for $30 \mathrm{~s}$. This was followed by 40 cycles of denaturation at $95^{\circ} \mathrm{C}$ for $10 \mathrm{~s}$, annealing at $62^{\circ} \mathrm{C}$ for $15 \mathrm{~s}$, amplification at $72^{\circ} \mathrm{C}$ for $8 \mathrm{~s}$ for 45 cycles. Cycles were followed by $30 \mathrm{~s} 40^{\circ} \mathrm{C}$ cooling program. The primers and probes were designed by the Light Cycler primer and probe set (Roche Diagnostics Applied Science, Tokyo). NOS-2, NOS-3 and NOS-1 primer sequences were designed on GenBank database NM_0100927, NM_008713, and NM_008712 respectively. All data were normalized by dividing with the corresponding GAPDH mRNA from the same sample.

Immunohistochemistry

Anesthetized animals were perfused intracardially with $20 \mathrm{ml}$ of $0.1 \mathrm{M}$ PBS, followed by $30 \mathrm{ml}$ of $4 \%$ paraformaldehyde in $0.1 \mathrm{M}$ phosphate buffer. The brains were removed, postfixed overnight and cryoprotected in 30\% sucrose, processed through graded alcohols and embedded in paraffin. Then, they were cut as $6 \mu \mathrm{m}$ thick coronal 
sections. They were incubated with $0.3 \% \mathrm{H}_{2} \mathrm{O}_{2}$ for $20 \mathrm{~min}$. The sections were then rinsed with tris-buffered saline, $\mathrm{pH}$ 7.6 (TBS). To identify reactive astrocytes, we stained with Anti-GFAP (rabbit polyclonal, DAKO, Denmark). Sections were incubated over night at a 1:2,000 dilution. The NOS-2 stains were incubated for $4 \mathrm{~h}$ at a 1:1,500 dilution (AntiiNOS/NOSII, rabbit polyclonal IgG;Upstate Biotechnology, Lake Placid, NY). After the first staining, each section was incubated with the secondary antibody for $30 \mathrm{~min}$ (Nichirei Histofine Simple Stain, anti-rabbit $\mathrm{IgG}$ ) at room temperature after TBS rinsing. Labeling was visualized by incubating with a solution containing $2 \%, 3,3^{\prime}$-diaminobenzidine, $0.1 \mathrm{M}$ imidazole and $30 \% \mathrm{H}_{2} \mathrm{O}_{2}$.

All sections were counterstained with Mayer's hematoxylin (SIGMA, MI).

\section{Statistical analysis}

Fisher's exact test was used when comparing body weights. All results obtained from the real time RT-PCR were subjected to one- or two-way analysis of variance (ANOVA) and differences among the means were analyzed by two-way ANOVA followed by a Newman-Keuls range test at the 0.05 significance level. Results are expressed as mean \pm standard error.

\section{Results}

\section{Body weight}

Figure 1 shows the change in body weight of the noninfected (PBS) and infected (Flu) mice over day. The mice infected with virus showed a decrease in weight and ruffled hair from 3 days p.i. and by 6 days p.i. and there was a significant decrease in the body weight of the infected mice as compared to the non-infected mice $(P<0.05)$. Prominent neurological signs in the infected mice did not appear during the experimental period and none of the mice died during the experiment, although spontaneous locomotor activity was reduced in the infected mice.

\section{Viral titers}

At one day p.i., $120 \mathrm{PFU} / 50 \mathrm{mg}$ virus was detected in the infected mouse brain and $136 \times 10^{4} \mathrm{PFU} / 100 \mathrm{mg}$ virus was detected in the infected mouse lung.

\section{Real-time RT-PCR for NOS-2, NOS-3 and NOS-1}

Figure 2 shows the NOS-2/GAPDH and NOS-3/GAPDH ratios as measured in the olfactory bulb and hippocampus

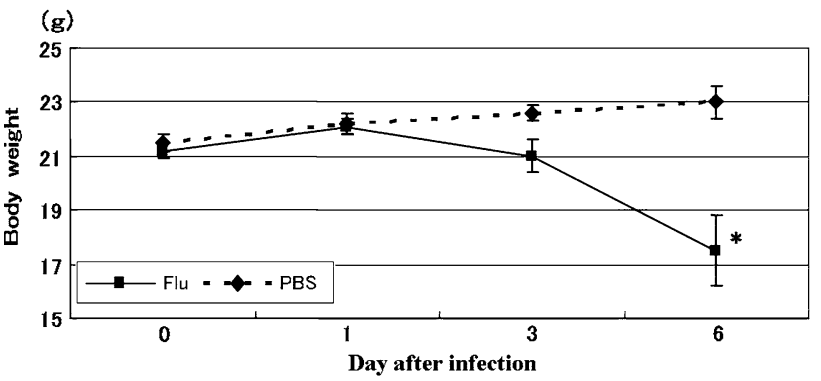

Fig. 1 Changes in body weight of A/NWS/33 influenza virus infected BALB/c model mice after virus infection. Each result shows the mean $\pm \mathrm{SE}$ value of eight experiments. (-) non-infected (PBS treatment) mice group, $(\cdot \bullet \cdot)$ infected (Flu) mice group

of infected mouse brains as compared to the non-infected mice on days 1,3 and 6 p.i. Due to the scattered results obtained in both the infected and non-infected mice, data on the NOS-1/GAPDH ratio is not reported. The increases in the NOS-2/GAPDH ratio as seen in the olfactory bul$\mathrm{b}(\mathrm{OB})$ and hippocampus(HIP) at day 1 and 3 p.i., were obvious as shown in Fig. 2a and b. However, no changes were seen in the levels of NOS-3/GAPDH in the same regions (Fig. 2a', b').

Changes in brain NO levels between non-infected and infected mice

Figure 3 shows that NO levels in the eight brain regions examined, either with or without NWS virus infection, changes in a day-by-day manner after virus infection. The levels of $\mathrm{NO}_{2}$ and $\mathrm{NO}_{3}$ were measured separately, as shown in the Materials and methods. In the isolated brain regions, $\mathrm{NO}_{3}$ levels were more than 40 -fold higher than $\mathrm{NO}_{2}$ levels, and the final data are shown as the total value (NO) of $\mathrm{NO}_{2}$ plus $\mathrm{NO}_{3}$. At day 1 p.i., the $\mathrm{NO}$ levels detected in olfactory bulb (OB), hippocampus (HIP) and frontal cortex (FC) of the infected mice (Flu) were significantly increased as compared to the levels in the same brain regions of the non-infected (PBS) mice $(P<0.05)$. Further, at day 6 p.i., the increased levels of $\mathrm{NO}$ in the olfactory bulb (OB) and the frontal cortex (FC) returned to the levels seen in non-infected mice. However, the levels of NO in the hippocampus (HIP) were significantly increased $(P<0.05)$ at all the time points examined.

Immunohistochemistry

Figure 4 shows the results of anti-NOS-2 staining and GFAP staining in the hippocampus of non-infected (PBS) and infected mice (Flu) at 1 (Fig. 4a) and 6 (Fig. 4b) days p.i. in the same magnification each. The brown-yellow 
Fig. 2 NOS-2/GAPDH and NOS-3/GAPDH ratios in the olfactory bulb and hippocampus of non-infected and infected mice on days 1,3 and 6 p.i. The open column shows the mean and S.E. value obtained from nine non-infected mice and the closed column shows the mean and S.E. value obtained from eight infected mice. *Significant difference between non-infected mice and infected mice. $* P<0.05$. (a and $\mathbf{a}$ '): olfactory bulb, (b and $\left.\mathbf{b}^{\prime}\right)$ : hippocampus
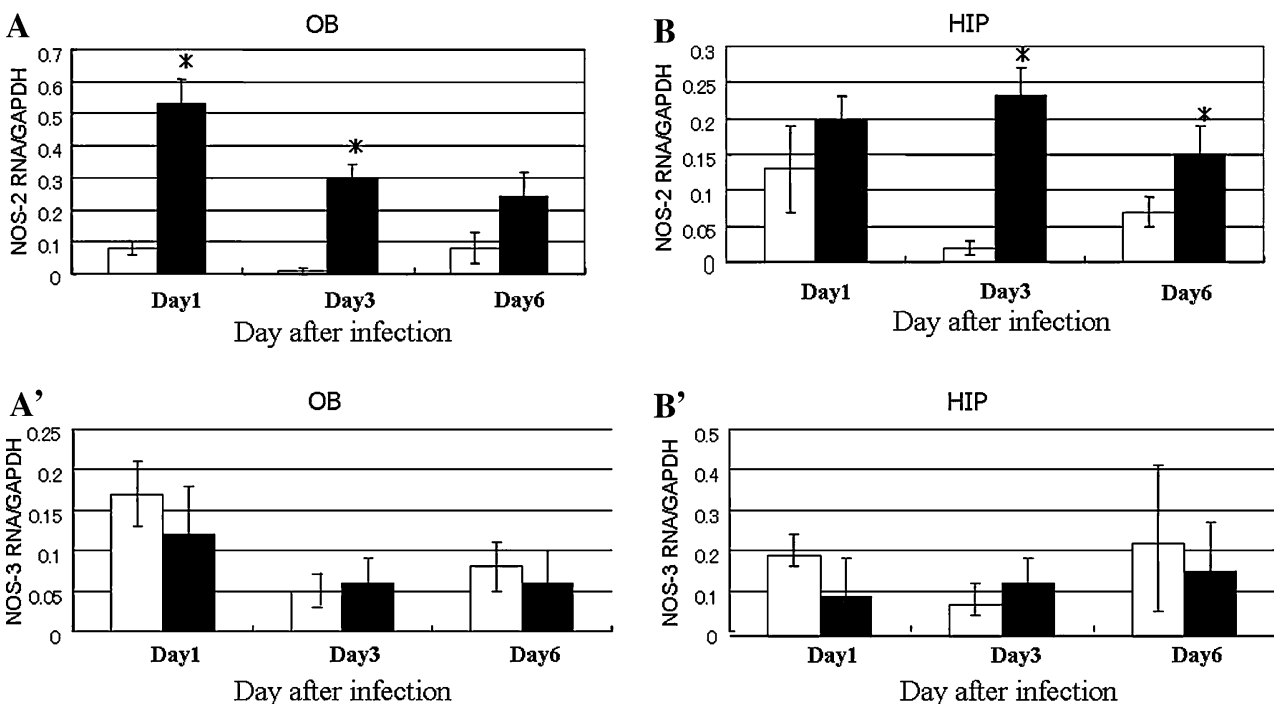
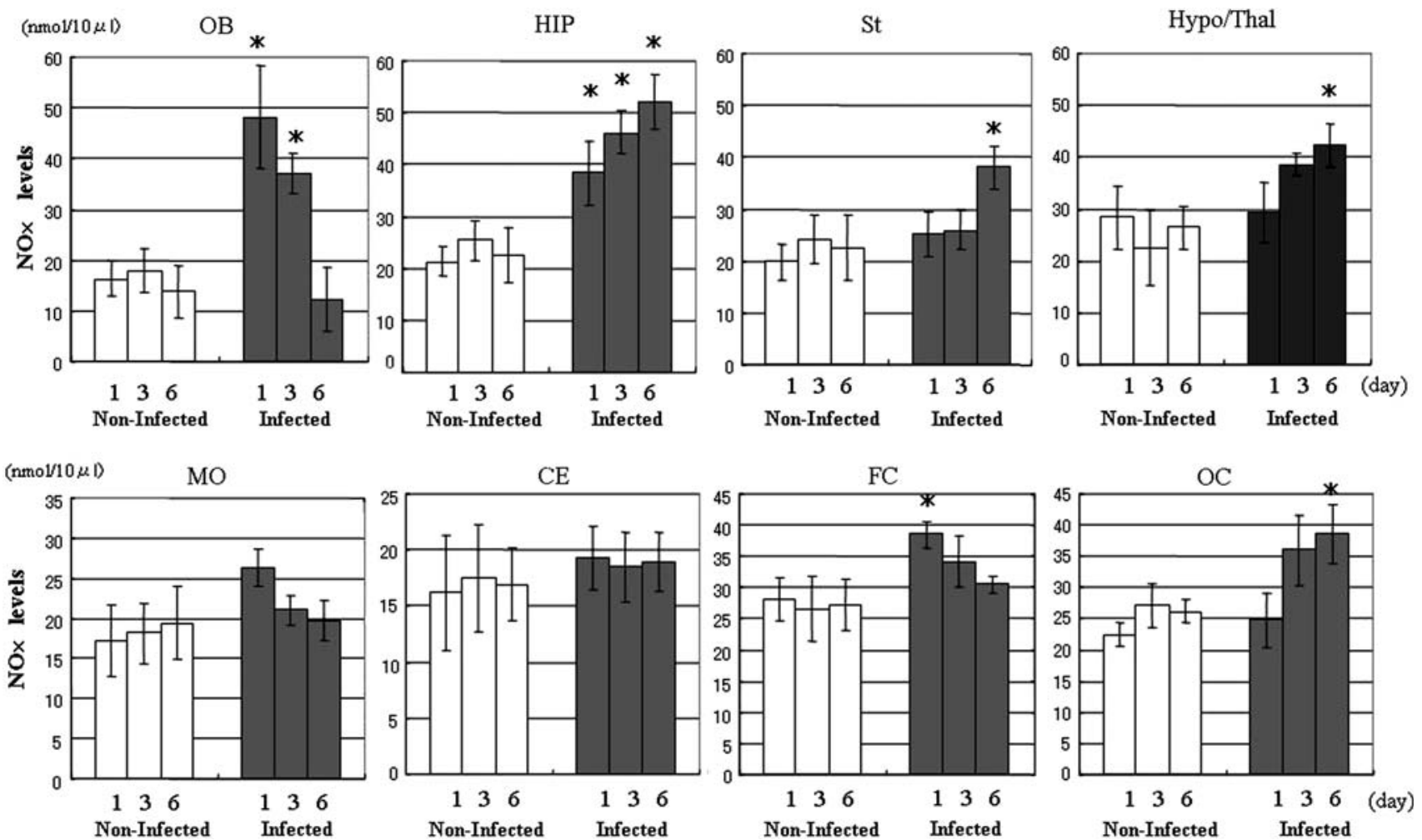

Fig. 3 Day to day changes in NO levels in the eight brain regions either with or without NWS virus infection. The open column shows the mean and S.E. value obtained from eight non-infected mice and the closed column shows the mean and S.E. value obtained from seven infected mice. *Significant difference between non-infected

staining was recognized as positive staining for anti-NOS2. We found positive staining in the hippocampus, especially in the area surrounding capillary blood vessels of the infected mice at 1 day p.i. (Fig. 4a, Day 1, Infected). By 6 days p.i., positive staining had spread to the striatum, hippocampus and cortex of the infected mice and positive

mice and infected mice. $* P<0.05$. OB: olfactory bulb, HIP: hippocampus, St: striatum, Hypo/Thal: hypothalamus and thalamus, MO: medulla oblongata, CE: cerebellum, FC: frontal cortex, and OC: occipital cortex

staining were seen in the areas surrounding capillary vessels (Fig. 4b, Day 6, Infected). Add that infected mice showed an increase in GFAP staining on days 1 much more so than that seen in non-infected mice. Positive staining was most notable around hippocampal blood vessels in infected mice. Additionally, most of the blood vessels in 
Fig. 4 Anti-NOS-2 immunohistochemistry and GFAP immunohistochemistry in the hippocampus at 1 (Fig. 4a) and 6 (Fig. 4b) days p.i

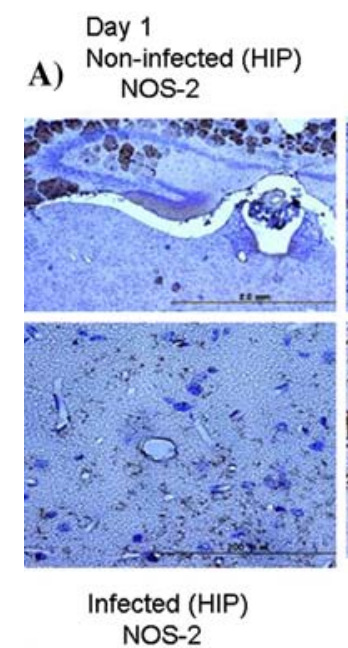

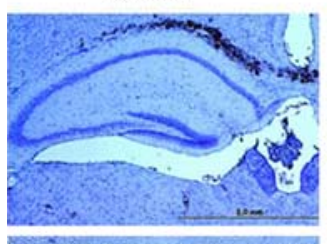

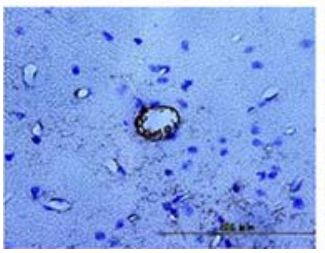

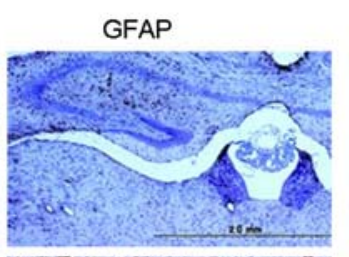
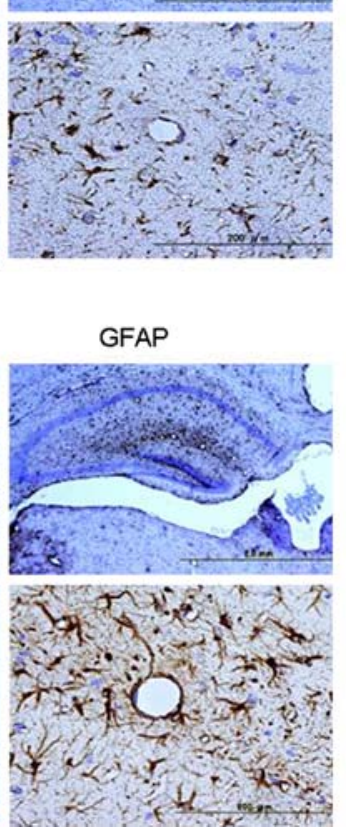

Day 6

B) $\begin{gathered}\text { Non-infected (HIP) } \\ \text { NOS-2 }\end{gathered}$
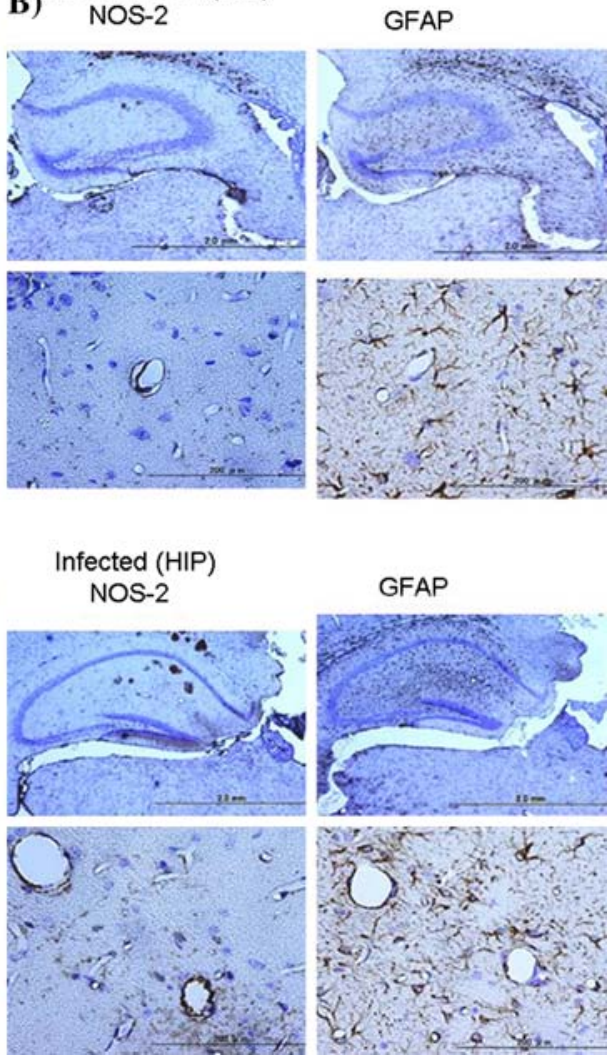

hippocampi of infected mice were markedly expanded as compared to those seen in the non-infected mice.

\section{Discussion}

Since our aim was to see the effect to the brain after influenza infection, this experiment was undertaken by using the $\mathrm{A} / \mathrm{NWS} / 33$ virus because it is proven by $\mathrm{Li}$ et al that the NWS/33 strain can replicate in the brain of adult mice after intracerebral injection, add that the virus usually fails to spread beyond the respiratory epithelium after internasal instillation [6].

During the experiment, we observed that the NWS virus infected mice lose significantly more body weight at 6 days p.i. (Fig. 1) in agreement with previous studies [5, 7]. Since bodyweight changes are an indicator of the overall condition of the mice, this bear out that the mice was infected to the virus. More over, we have checked that RT-PCR for A/ NWS/33 virus were positive in all brain regions expect medullar oblongata on day 1 p.i. (data not shown).

The reason why we determined the amounts of influenza virus in this experiment is as followed. During the past experiment, we had experienced that the mice die within 3 days p.i. when inoculating any more than the virus we used this time and when we use fewer viruses, there were hardly any difference in the non-infected and infected behavioral change.

Our results show that an enhancement NO biosynthesis was induced in the NWS virus infected mouse brain. Overproduction of NO was substantiated by determining an increase in the expression of NOS-2 mRNA as shown in Fig. 2, and increased immuno-histochemical staining for anti-NOS-2 as shown in Fig. 4. At 1 day p.i., the amount of NOS-2 mRNA within the infected mouse brain was greater than that seen in control mice in all brain regions studied. Above all, the olfactory bulb showed the greatest increase. On the basis of these results, we compared NOS-2 mRNA expression in the olfactory bulb on days 1,3 , and 6 p.i., respectively. This showed that the NOS-2 mRNA ratio was greater in the infected mouse brain at all experimental times studied (Fig. 2). On the contrary, there was no difference in NOS-3 or NOS-1 mRNA levels between infected and control mice (data not shown). These results are important since these three isoforms of NOS, NOS-2 the inducible form of NOS, is capable of producing a continuous flux of $\mathrm{NO}$ and a large amount of $\mathrm{NO}$ generated by NOS-2 over a sustained period is implicated in the pathogenesis of various disorders and with the response to infectious organisms [8]. 
In regards to the immuno-histochemical analysis, positive staining for anti-NOS-2 was mostly detected in the hippocampus of infected mouse brains, whereas hardly any staining was seen in control brains (Fig. 4). Furthermore, positive staining had spread to the hippocampus and striatum by 6 days p.i., which correlated with the transition of NO levels.

NOS-2 has been shown to play a role in the defense against bacteria, parasites and viruses. However, it seems that NO induced by the influenza virus has a dual role. Rimmelzwaan et al. reported an inhibitory effect of NO on virus growth [9], whereas Akaike et al. reported that NO played a pathological role in the influenza virus-induced pneumonia in mice [10]. The fact that the influenza virus can induce NO has been reported previously [2, 3]. Yao et al. reported that the mRNA's of both NOS-2 and NOS increased in the brain after the intranasal infection with influenza A virus of newborn mice [11]. Further, Imanishi et al. pointed out that a mouse lung-adapted strain of influenza virus could induce NOS-2 mRNA expression and NOS-2-mediated NO production in murine macrophage cells [5]. Our study has demonstrated that the NWS virus can induce NOS-2 expression in the infected mouse brain, especially in the olfactory bulb and hippocampus. These results may relate to the fact that intranasally administered NWS virus can invade the brain through the olfactory pathway as has been reported previously [12-16]. Recently, Yossi et al. reported that astrocytes are capable of rapid and massive NOS-2-dependent NO production in brains of normal mice in situ [17]. According to the report by Ino et al. on the brain autopsy of patients who had died of IE, four out of five IE patients showed degenerated astrocytic spines in the cortex by GFAP staining. They suspected that the IE was largely due to altered glial cells (Ino et al. unpublished). Takahashi et al. also made a statement on the autopsied case of a 2-year-old girl with encephalopathy associated with influenza A virus infection and according to their report; reactive astrocytes were seen in the vicinity of blood vessel in the brain but astrocytosis was not seen anywhere, including the areas where virus antigen-positive neurons were abundant [18]. Additionally, Yokota et al. hypothesized that the influenza virus activates the limbic system followed by the activation of glial cells which produce NO and other inflammatory cytokines which together flood the brain and cause a cytokine storm [4]. Astrocytes are the major glial cell in the CNS and have important physiological properties in terms of CNS homeostasis. Astrocytes also influence the formation and maintenance of the blood-brain barrier (BBB). The importance of astrocytes is demonstrated by a study from Bush et al. According to their report, an astrocyte ablation led to failure of BBB repair and vasogenic edema [19]. There have been several reports related to glial cells and
NO [17, 20, 21]. However, there has not been a report considering the relationship between influenza virus infection, NOS-2 and astrocytes together. By determining an increase in GFAP staining as shown in Fig. 4, our results show that astrocytes are activated in the infected mouse brain and especially in hippocampus. Interestingly, we noticed that both the increased NOS-2 and GFAP staining were mostly found around capillary blood vessels of the hippocampus starting at an early stage. Our results suggest that since NOS-2 and astrocytes became activated around capillary blood vessels after viral infection, this may lead to brain capillary blood vessels break down. However, we were unable to determine whether the NOS-2 measured is derived from vascular endothelial cells or macrophages which will be an issue for future experiments.

Although IE has been mostly reported in Asian countries, influenza infection occurs in epidemics in both the northern and southern hemispheres and is a pandemic disease throughout the year. Recently, influenza virus which is resistant to anti-influenza drugs has been shown to emerge and there is real concern in regards to a new type of influenza pandemic [22]. However, no standardized therapy for the treatment of IE has yet been defined. In this paper, we suggest that the influenza virus causes the activation of astrocytes and NOS-2 in the brain. Thus it is likely that our results will add supportive evidence for the development of a novel IE therapy.

Acknowledgements The authors gratefully acknowledge the kind donation of influenza A/NWS/33 virus by Prof. Hayashi (Toyama University, School of Pharmaceutical Sciences) and active discussions throughout this study with Prof. J. Yamada (Tokyo Medical University, Department of Anatomy). This work was supported by Japan Society for the Promotion of Science, Grant-in-Aid for Scientific Research B (Y.W.) and C (H.K.).

Open Access This article is distributed under the terms of the Creative Commons Attribution Noncommercial License which permits any noncommercial use, distribution, and reproduction in any medium, provided the original author(s) and source are credited.

\section{Reference}

1. Morishima T, Togashi T, Yokota S et al (2002) Encephalitis and encephalopathy associated with an influenza epidemic in Japan. Clin Infect Dis 35:512-517

2. Kawashima H, Watanabe Y, Ichiyama T et al (2002) High concentration of serum nitrite/nitrate obtained from patients with influenza-associated encephalopathy. Pediatr Int 44:705-707

3. Kawashima H, Amaha M, Ioi H et al (2005) Nitrite/nitrate (NOx) and zinc concentrations in influenza-associated encephalopathy in children with different sequela. Neurochem Res 30(3):311-314

4. Yokota S, Kobayashi Y, Nezu A (2005) Cytokine therapy. Clin Neurosci 23:931-934 (Abstract in English)

5. Imanishi N, Andoh T, Sakai S et al (2005) Induction of inducible nitric oxide (NO) synthase mRNA and NO production in 
macrophages infected with influenza $\mathrm{A} / \mathrm{PR} / 8$ virus and stimulated with its ether-split product. Microbiol Immunol 49(1):41-48

6. Li S, Schulman J, Itamura S et al (1993) Glycosylation of neuraminidase determines the neurovirulence of influenza A/NSW/33 virus. J Virol 67:6667-6673

7. Shinjoh M, Yoshikawa T, Li Y et al (2002) Prophylaxis and treatment of influenza encephalitis in an experimental mouse model. J Med Virol 67:406-417

8. Nathan C (1997) Inducible nitric oxide synthase. J Clin Invest 100:2417-2423

9. Rimmelzwaan GF, Baars MMJW, Lijster P et al (1999) Inhibition of influenza virus replication by nitric oxide. J Virol 73(10):8880-8883

10. Akaike T, Noguchi Y, Ijiri S et al (1996) Pathogenesis of influenza virus induced pneumonia: involvement of both nitric oxide and oxygen radicals. Proc Natl Acad Sci USA 91:10089-10093

11. Yao D, Kuwajima M, Kido H (2003) Pathologic mechanism of influenza encephalitis with an abnormal expression of inflammatory cytokines and accumulation of mini plasmin. J Med Invest 50:1-8

12. Matuda K, Park C, Sunden Y et al (2004) The vagus nerve is one route of transneural invasion for intranasally inoculated influenza A virus in mice. Vet Pathol 41:101-107

13. Reinacher M, Bonin J, Narayan O et al (1983) Pathogenesis of neurovirulent influenza A virus infection in mice. Lab Invest 49(6):686-692

14. Shinya K, Shimada A, Ito T et al (2000) Avian influenza virus intranasally inoculated infects the central nervous system of mice through the general visceral afferent nerve. Arch Virol 145:187195

15. Park C, Ishinaka M, Takada A et al (2002) The invasion routs of neurovirulent A/Hong Kong/483/97(H5N1) influenza virus into the central nervous system after respiratory infection in mice. Arch Virol 147:1425-1436

16. Aronsson F, Robertson B, Ljunggren $H$ et al (2003) Invasion and persistence of the neuroadapted influenza virus $\mathrm{A} / \mathrm{WSN} / 33$ in the mouse olfactory system. Viral Immunol 16(3):415-423

17. Yossi B, Shai F, Michal H et al (2005) Rapid and reactive nitric oxide production by astrocytes in mouse neocortical slices. Glia 52:169-176

18. Takahashi M, Yamada T, Nakashita Y et al (2000) Influenza virus-induced encephalopathy: clinicopathologic study of an autopsied case. Pediatr Int 42:204-214

19. Toby GB, Narman P, Catherine HH et al (1999) Leukocyte infiltration, neuronal degeneration, and neurite outgrowth after ablation of scan-forming, reactive astrocytes in adult transgenic mice. Neuron 23:297-308

20. Murphy S (2000) Production of nitric oxide by glial cells: regulation and potential roles in the CNS. Glia 29:1-14

21. Brahmachari S, Fung Y, Pahan K (2006) Induction of glia fibrillary acidic protein expression in astrocytes by nitric oxide. $\mathrm{J}$ Neurosci 26(18):4930-4939

22. Hatakeyama S, Kawaoka Y (2006) The molecular basis of resistance to anti-influenza drugs. Nippon Rinsho 64(10):18451851 (Abstract in English) 